\title{
LAS MEDICINAS ALTERNATIVAS Y COMPLEMENTARIAS COMO RECURSO EN LOS ITINERARIOS TERAPÉUTICOS DE LAS MUJERES. IMPORTANCIA EN NUESTRO CONTEXTO
}

\author{
ENRIQUE PERDIGUERO \\ BEATRIZ TOSAL \\ Universidad Miguel Hernández (Elche)
}

\section{INTRODUCCIÓN}

Existen diferencias entre mujeres y hombres tanto en los patrones de mortalidad y morbilidad y en la percepción de los síntomas como en la gestión de sus problemas de salud. En el caso de la utilización de servicios sanitarios, se ha constatado que las mujeres son más frecuentadoras que los hombres de todos los servicios de salud, pero especialmente de los servicios de atención primaria ${ }^{1}$.

Se han propuesto múltiples explicaciones para esta situación, entre las que se encuentran, por ejemplo, la mayor consciencia que sobre la salud tienen las mujeres, lo que les conduce a la utilización de recursos relacionados con la promoción de la salud y la prevención de las enfermedades, la influencia de su rol doméstico como cuidadoras informales que las convierte en más 'expertas' en los temas relacionados con la salud, su mayor sensibilidad ante la presencia de determinados signos y síntomas, su mayor morbilidad relacionada con su

1. GReEn, C.A.; PoPe, C.R.: "Gender, psychosocial factors and the use of medical services», Social Science and Medicine, 48 (1999), pp. 1363-1372. BerTaKis, K.D.; AZARI, R.; HeLmS, L.J.; CALlaHAN, E.J.; RoBBINS, J.A.: "Gender differences in the utilization of health care services», Journal of Family Practice, 49 (2000), pp. 147-152. Redondo, A.; Guallar, P.; BANEGAS, J.R.; RodRIGUeZ, F.: "Gender differences in the utilization of health-care services among the older population of Spain", BMC Public Health, 6 (2006), p. 155 (http://www.pubmedcentral.nih.gov/articlerender. fcgi?artid=1569836). 
posición social y los roles sociales que desempeñan, o su mayor facilidad para aceptar ayuda y cumplir con los tratamientos prescritos ${ }^{2}$.

Estas diferencias que obedecen, como podemos observar, a múltiples causas de tipo biológico, psicológico, social y cultural, han sido evidenciadas, también, en cuanto al tipo de instancias terapéuticas que se utilizan para resolver los problemas de salud. Existe consenso en la apreciación de que las mujeres son las mayores usuarias no sólo de los servicios sanitarios sino también de las medicinas alternativas y complementarias (a partir de este momento MAC) para resolver sus problemas de salud ${ }^{3}$.

La importancia que el fenómeno de las MAC tiene en los países más ricos ha reavivado el interés por el estudio del pluralismo asistencial y terapéutico ${ }^{4}$, y sirve para constatar que la resolución de los problemas de salud no es un asunto que se maneje sólo a través del uso de servicios sanitarios, lo que ha puesto en

2. Existe una extensa producción bibliográfica sobre las diferencias en la utilización de servicios sanitarios por parte de hombres y mujeres y las hipótesis sobre las causas de dichas diferencias. Entre los trabajos más frecuentemente citados se encuentran: ClEARY, P.D.; MECHANIC, D.; GREENLEY, J.R. "Sex differences in medical care utilization: an empirical investigation», Journal of Health and Social Behavior, 23 (1982), pp. 106-119. VerbrUGGe, L.M.: "Gender and Health: an update on hypotheses and evidence", Journal of Health and Social Behavior, 26 (1985), pp. 156-182. HIBBARD, J.H.; POPE, C.R.: «Another look at sex difference in the use of medical care: illness orientation and the types of morbidities for which services are used", Women and Health, 11 (1986), pp. 21-36. Gijsbers VAn WijK, C.M.T.; VAn Vliet, K.P; KolK, A.M.; EVeraerd, W.: «Symptom sensitivity and sex differences: a review of health surveys in the United States and the Netherlands", Women and Health, 17 (1991), pp. 91-124. Anson, O.; PARAN, E.; NEUMANN, L.; CHERNICHOVSKY, D.: "Gender differences in health perceptions and their predictors", Social Science and Medicine, 36 (1993), pp. 419-427. MACINTYRE, S.; HUNT, K.; SWEETING, H.: «Gender differences in health: are things really as simple as they seem?», Social Science and Medicine, 42 (1996), pp. 617-624.

3. Kessler, R.C.; DAVIS, R.B.; Foster, D.F.; VAn Rompay, M.I.; Walters, E.S.; WillKey, S.J.; KaPCHUCK, T.J.; EISENBERG, D.M.: "Long-Term Trends in the Use of Complementary and Alternative Medical Therapies in the United States", Annals of Internal Medicine, 135 (2001), pp. 262-268. Complementary and Alternative Medicine in the United States, Washington, The National Academies Press, 2005. Johannessen, H.; LÁZÁr, I. (eds.): Multiple Medical Realities. Patients and Healers in Biomedical, Alternative and Traditional Medicine, New York-Oxford, Berhghan Books, 2006.

4. Entendemos por pluralismo asistencial la existencia en una sociedad dada de diversas instancias asistenciales y terapéuticas que son utilizadas por sus miembros para resolver sus problemas de salud. La definición y valoración de los propios problemas de salud y su relación con el contexto social económico, político y cultural entra, por supuesto, en el ámbito de estudio del pluralismo asistencial. Según las tradiciones académicas el conocimiento que se tiene de este fenómeno es muy desigual. Los estudios generados por los propios profesionales sanitarios, por la sociología de la salud anglosajona, o por la psicología social, al adoptar en muchas ocasiones una perspectiva basada en el uso de servicios (habitualmente de las diferentes instancias asistenciales que ofrece la medicina científica-occidental) han dejado fuera de foco gran parte de lo que la población hace habitualmente para recuperar su salud. Y lo que es más importante, han transformado ciertos modelos de descripción del comportamiento de la enfermedad, en modelos 'prescriptivos' de tal comportamiento. Perdiguero, E.: «Una reflexión sobre el pluralismo médico», en G. Fernández Juárez (coord): Salud e Interculturalidad en América Latina. Antropología de la Salud y Crítica Intercultural, Quito, Abya-Yala - Universidad de Castilla La Mancha, 2006, pp. 33-49. YounG, J.T.: «Illness behaviour: a selective review and synthesis», Sociology of Health and Illness, 26 (2004), pp.1-31. MACKIAN, S.; BEDRI, N.; LOVEL, H.: «Up the garden path and over the edge: where might health-seeking behaviour take us?», Health Policy and Planning, 19 (2004), pp. 137-146. 
tela de juicio los modelos 'prescriptivos' de comportamiento frente a la enfermedad y ha colocado sobre el tapete el tema del pluralismo asistencial como fenómeno estructural, en circuitos académicos y sociales que no lo reconocían como tal ${ }^{5}$.

En todo caso las MAC sólo son la parte que recientemente se ha hecho más visible del pluralismo asistencial, junto con la automedicación -bien documentada en todas las encuestas de salud realizadas en los últimos años ${ }^{6}$, aunque el concepto hace referencia a otras muchas instancias terapéuticas entre las que se encuentran desde las diversas formas de autoatención ${ }^{7}$, además de la automedicación, hasta el recurso a redes sociales más o menos formales como grupos de ayuda mutua o asociaciones de enfermos.

\section{EL USO CRECIENTE DE LAS MAC}

Las medicinas complementarias y alternativas han recibido en los últimos lustros una atención creciente, tanto en el entorno de la Unión Europea como en Norteamérica y Oceanía ${ }^{8}$. No sólo se han llevado a cabo estudios en los países más ricos, sino, también, otros centrados en otros ámbitos del planeta?. Este interés ha sido generado por la constatación del uso creciente de las MAC en las sociedades en las que la medicina científica-occidental se encuentra más desarrollada.

Uno de los principales obstáculos para medir la prevalencia de uso de las $\mathrm{MAC}$ es la dificultad de definir, precisamente, lo que entendemos por tales

5. GoldSTEIN, M.S.: "The persistence and Resurgence of Medical Pluralism», Journal of Health Politics, Policy and Law, 29 (2004), pp. 925-945.

6. Se pueden consultar en http://www.msc.es/estadEstudios/estadisticas/encuestaNacional/home. htm

7. El concepto de autoatención hace referencia a las diversas «representaciones y prácticas que la población utiliza a nivel de sujeto y grupo social para diagnosticar, explicar, atender, controlar aliviar, aguantar, curar, solucionar o prevenir los procesos que afectan a su salud (...) sin la intervención central, directa e intencional de curadores profesionales, aun cuando estos puedan ser la referencia de la actividad de autoatención; implica decidir la autoprescripción y el uso de un tratamiento en forma autónoma.» MENENDEZ, E. «Autoatención y automedicación. Un sistema de transacciones sociales permanentes», en Antropología Médica. Orientaciones, desigualdades y transacciones, México, Cuadernos de la Casa Chata, 1990, pp. 165-204.

8. Salmon, J.W.: Alternative Medicines. Popular and policy perspectives, New York-London, Tavistock, 1984. LeWITH, G.; ALDRIDGE, D.: Complementary medicine and the European Community, Cambridge, Saffron, 1991. Walden, C.W.; SHARMA, U.: Complementary Medicine Today, London, Routledge, 1992. FISHER, P.; WARD, A.: «Medicine in Europe: Complementary medicine in Europe», British Medical Journal, 309 (1994), pp. 107-111. CANT, S.; SHARMA, U. (eds.): Complementary and Alternative Medicines. Knowledge in Practice, London and New York, Free Books, 1996. KeLneR, M.J.; Wellman, B.; Pescosolido, B.; SAKS, M. (eds.): Complementary and Alternative Medicine: Challenge and Change, Amsterdam, Gordon and Breach, 2000. TOVEY, P.; EASTHOPE, G.; ADAMS, J. (eds.): The mainstreaming of complementary and alternative medicine: studies in social context, LondonNew York, Routledge, 2003. RugGy, M.: Marginal to Mainstream. Alternative Medicine in America, New York, Cambridge Univesity Press, 2004.

9. Bodeker, G.; KronenberG, F.: «A Public Health Agenda for Traditional, Complementary, and Alternative Medicine», American Journal of Public Health, 92 (2002), pp. 1582-1591. 
medicinas ${ }^{10}$. La clarificación de qué formas de sanar deben ser consideradas como tales es un asunto ampliamente discutido, pero resulta muy difícil llegar a un consenso, dado que bajo esta etiqueta se consideran tanto formas de sanar milenarias con tradiciones doctrinales bien establecidas como modas dietéticas de efímera vida ${ }^{11}$.

Otro punto de difícil acuerdo se centra en si se debe incluir en el concepto de 'uso' el consumo de productos como complejos vitamínicos o hierbas o tan sólo el hecho de acudir a un proveedor de MAC. La decisión sobre este asunto explica no pocas de las discrepancias encontradas en la prevalencia de uso de las MAC, incluso dentro del mismo país, tal y como ocurre en los Estados Unidos de América, con cifras muy variables que van desde el 6,5\% al 42,1\%. Aunque los datos parecen apuntar con consistencia a que un tercio de la población las utiliza de una u otra forma ${ }^{12}$.

Por lo que hasta ahora se sabe no es aventurado afirmar que, en la actualidad, en muchos contextos las MAC se usan frecuentemente y con una tendencia creciente, si bien es difícil valorar de manera exacta el grado en el que la población recurre a ellas. Desde luego su utilización varía en función del género y de la clase social. Especialmente recurren a ellas mujeres con educación media o superior y con niveles de ingresos medios o altos.

En cuanto a las MAC más usadas varían mucho según los contextos investigados. Los motivos que llevan a la población a utilizar este tipo de opciones es uno de los problemas más interesantes planteados hoy día para el que no hay una respuesta clara todavía ${ }^{13}$. Tiene que ver desde luego con el cambio del patrón epidemiológico en las sociedades más opulentas, plagadas de padecimientos crónicos con los que hay que convivir; con un cierto rechazo del consumo de productos químicos, aunque esto no debe hacernos pensar en que la población mira la ciencia y la técnica con desconfianza; y, en gran medida, con la transformación de todo lo relacionado con la salud en un objeto de consumo más ${ }^{14}$.

10. KaptchuK, T.J.; EisenberG, D.M.: «Varieties of Healing. 1: Medical Pluralism in the United States», Annals of Internal Medicine, 135 (2001), pp. 189-195. KAPTCHUK, T.J.; EISENBERG, D.M.: "Varieties of Healing. 2: A Taxonomy of Unconventional Healing Practices», Annals of Internal Medicine, 135 (2001), pp. 196-204.

11. Así, por ejemplo, un reciente artículo sobre el uso de las MAC en los Estados Unidos de América eleva al $62 \%$ el porcentaje de mayores de 18 años que las usaron en el año previo si se considera dentro de estas medicinas la oración con objetivo terapéutico o paliativo. Este porcentaje desciende a un $36 \%$ cuando esta se excluye. BARNES, P.M.; POWELL-GRINER, E.; MACFANN, K.; NAHIN, R.L.: «Complementary and alternative medicine use among adults: United States, 2002», Advance Data, 27 (2004), pp. 1-19.

12. NI, H.; Simile, C.; HARDY, A.M.: «Utilization of complementary and alternative medicine by United States adults: results from the 1999 national health interview survey", Medical Care, 40 (2002), pp. 353-358.

13. COULTER, I.D.; WiLLIS, E.M.: "The rise and rise of complementary medicine: a sociological perspective", Medical Journal of Australia, 180 (2004), pp. 587-589.

14. Doel, M.A.; SEGROTT, J.: "Beyond belief? Consumer culture, complementary medicine, and the dis-ease o everyday life», Enviroment and Planning D: Society and Space, 21 (2003), pp. 739-759. 
Como respuesta a esta creciente tendencia de uso y a su repercusión económica ${ }^{15}$ diversos gobiernos como, por ejemplo, los de Canadá (1999), Reino Unido (2002) y Estados Unidos (2002) han publicado informes oficiales. En ellos se trata de ir avanzando en variados aspectos como la financiación de las MAC, la acreditación de los proveedores de las mismas, la formación de los profesionales sanitarios en estas terapias o la eficacia y efectividad de las mismas ${ }^{16}$.

\section{LAS DIFICULTADES DE DEFINICIÓN DE LAS MAC}

Como ya poníamos de manifiesto en el apartado anterior, existen muchas dificultades para conocer la realidad de las medicinas alternativas y complementarias.

El principal obstáculo tiene que ver con el problema de definición de lo que son realmente las MAC, y la relación de éstas con lo que se suele denominar medicina tradicional. Prácticamente no hay escrito que aborde con seriedad el tema de las MAC que no señale la dificultad que existe a la hora de saber qué podemos considerar como medicinas alternativas y complementarias. Los esfuerzos de clarificación siguen siendo frecuentes en la literatura internacional ${ }^{17}$, sin que se consiga avanzar mucho. Dada la situación de dominio de la medicina norteamericana en el ámbito internacional, la definición más citada es la del National Center for Complementary and Alternative Medicine (NCCAM): «[...] un conjunto diverso de sistemas, prácticas y productos médicos y de atención de la salud que no se consideran actualmente parte de la medicina convencional $[\ldots]{ }^{18}$.

A partir de esta definición se intenta clasificar a todas las MAC en una serie de grupos en función del desarrollo del cuerpo de conocimientos en que se sustentan y las técnicas que emplean para el restablecimiento de la salud, como se observa en la siguiente tabla:

15. El grupo de Harvard capitaneado por Eisenberg estimó un aumento del $45 \%$ en el gasto en proveedores de medicinas alternativas entre 1990 y 1997, y en Australia se ha encontrado un aumento de un $120 \%$ en el gasto en medicaciones alternativas entre 1993 y el 2000. MACLENNAN, A.H.; WILSON, D.H.; TAYLOR, A.W.: «The escalating cost and prevalence of alternative medicine», Preventive Medicine, 35 (2002), pp. 166-173.

16. Herman, P.M.; Craig, B.M.; CASPI, O.: «Is complementary and alternative medicine (CAM) cost-effective? A systematic review», BMC Complementary and Alternative Medicine, 5 (2005), p. 11.

17. Baldwin, C.M.; Kroesen, K.; Trochim, W.M.; Bell, I.R.: «Complementary and conventional medicine: a concept map", BMC Complementary and Alternative Medicine, 4 (2004), p. 2 (http:// www.pubmedcentral.nih.gov/articlerender.fcgi?artid=356920).

18. National Center for Complementary and Alternative Medicine. D-164. "¿Qué es la medicina complementaria y alternativa?», Bethesda, Maryland, Nacional Institutes of Health, Disponible en: http://nccam.nih.gov/espanol/informaciongeneral/ 
Clasificación de los grupos de MAC (NCCAM)

\begin{tabular}{|c|c|}
\hline GRUPOS DE MAC & EJEMPLOS \\
\hline Sistemas médicos alternativos & $\begin{array}{l}\text { Homeopatía } \\
\text { Naturopatía } \\
\text { Medicina Tradicional China } \\
\text { Medicina Ayurveda }\end{array}$ \\
\hline Enfoque sobre la mente y el cuerpo & $\begin{array}{l}\text { Meditación } \\
\text { Oración } \\
\text { Curación mental } \\
\text { Otras: } \\
\text { - Danza } \\
\text { - Música } \\
\text { - Arte }\end{array}$ \\
\hline Terapias biológicas & $\begin{array}{l}\text { Suplementos dietéticos } \\
\text { Productos de herboristería } \\
\text { Otras terapias 'naturales' (cartílago de tiburón) }\end{array}$ \\
\hline $\begin{array}{l}\text { Métodos de manipulación y basados en el } \\
\text { cuerpo }\end{array}$ & $\begin{array}{l}\text { Quiropráctica } \\
\text { Osteopatía } \\
\text { Masaje }\end{array}$ \\
\hline Terapias sobre la base de la energía & $\begin{array}{l}\text { Terapias del biocampo: } \\
\text { - Chi gong } \\
\text { - Reiki } \\
\text { - Toque terapéutico } \\
\text { Terapias bioelectromagnéticas (uso de): } \\
\text { - Campos de impulsos } \\
\text { - Campos magnéticos } \\
\text { - Campos de corriente alterna o directa }\end{array}$ \\
\hline
\end{tabular}

Como podemos comprobar todas las medicinas que se apartan de las que el NCCAM considera convencional en Estados Unidos son consideradas MAC, independientemente de cuál sea su situación de hegemonía en otros ámbitos, como ocurre con algunas de las que el NCCAM considera 'sistemas médicos alternativos'.

La definición utilizada por el NCCAM, no obstante, ha sido criticada desde los propios Estados Unidos de América, poniendo de manifiesto las dificultades intrínsecas de la etiqueta MAC, un producto histórico recién llegado, que al definirse en relación a una medicina científica-occidental, que es tomada por dominante, nunca podrá mostrar ninguna congruencia interna. Así el informe Complementary and Alternative Medicine in the United States publicado por The Nacional Academy of Sciences discute con detenimiento el tema ${ }^{19}$ opinando que la definición del NCCAM resulta inconsistente e insuficiente ${ }^{20}$. Siempre habrá

19. Complementary and Alternative Medicine in the United States, Op. cit., pp. 16-20.

20. El informe propone la siguiente definición: «Complementary and alternative medicine (CAM) is a broad domain of resources that encompasses health systems, modalities, and practices and their accompanying theories and beliefs, other than those intrinsic to the dominant health systems of a particular society or culture in a given historical period. CAM includes such resources perceived by their uses as associated with positive health outcomes. Boundaries within CAM and between the CAM domain and the domain of the dominant system are not always sharp or fixed» (Ibíd., p. 19). 
casos de determinadas sociedades con una hegemonía mucho más discutible de la medicina científica-occidental en las que algunas de las MAC enumeradas en la clasificación elaborada por el NCCAM no sean ni alternativas ni complementarias sino las medicinas dominantes de esa sociedad.

A esta confusión terminológica han contribuido también los organismos internacionales. La O.M.S. optó, años atrás, por la etiqueta 'medicina tradicional' como:

"[...] término amplio para referirse tanto a los sistemas de medicina tradicional como por ejemplo la medicina tradicional china, el ayurveda hindú y la medicina unani árabe, y a las diversas formas de medicina indígena. Las terapias de la medicina tradicional incluyen terapias con medicación, si implican el uso de medicinas con base de hierbas, partes de animales y/o minerales, y terapias sin medicación, si se realizan principalmente sin el uso de medicación, como en el caso de la acupuntura, las terapias manuales y las terapias espirituales [...]» ${ }^{21}$

Como puede comprobarse la medicina tradicional, tal y como la consideró la O.M.S, es tan comprehensiva como las MAC y con el mismo referente: todo lo que no es medicina científico-occidental.

Pero la creciente importancia que las denominadas MAC están teniendo en el mundo más desarrollado ha modificado algo el énfasis de la O.M.S. en la medicina tradicional y la etiqueta MAC está apareciendo con mayor fuerza en los últimos años ${ }^{22}$. En todo caso lo que parece existir es una tendencia a llamar a las mismas formas de asistir y sanar MAC cuando nos estamos refiriendo a ellas en el ámbito de los países más pudientes, en los que la hegemonía de la medicina científico-occidental es indiscutible, y medicinas tradicionales cuando nos estamos refiriendo a ellas en ámbitos en los que la hegemonía de la medicina científico-occidental resulta mucho más débil. Todo ello lleva a dificultades terminológicas, que en nuestro caso van a obstaculizar en gran medida el estudio del papel de otras opciones diferentes a la medicina científico-occidental en los itinerarios terapéuticos de las mujeres.

\section{LAS MAC EN ESPAÑA}

Aunque la caracterización de la realidad de las medicinas alternativas y complementarias en España esta todavía lejos de alcanzar el nivel de conocimiento que se da en otros ámbitos, es cierto que en los últimos años la situación está cambiando rápidamente.

Un primer indicador es el cambio en la consideración que muestran las revistas científicas de ciencias de la salud sobre las MAC. Si bien en España no contamos con una caracterización de la atención que se presta a las MAC en las publicaciones periódicas médicas -similar a las que se han publicado sobre los

21. ORGANIZACIÓN MUNDIAL DE LA SALUD: Estrategia de la OMS sobre medicina tradicional 2002-2005, Ginebra, OMS, 2002.

22. World Health Organization: WHO Medicines Strategy. Countries at the Core 2004-2007, Ginebra, WHO, 2004. 
Estados Unidos $^{23}$-, no parece aventurado señalar que las MAC están siendo valoradas de manera algo diferente por los profesionales sanitarios en los últimos años. Hace tan sólo una década no era nada extraño encontrar descalificaciones apriorísticas de las MAC, sin prestar demasiada atención a la importancia que como instancias asistenciales estaban adquiriendo en nuestro contexto económico y social, la Unión Europea ${ }^{24}$. Sin embargo, en los últimos años, son mucho más frecuentes los artículos que, cuanto menos, hacen una valoración más matizada de las $\mathrm{MAC}^{25}$, aunque persisten las dudas sobre su validez terapéutica ${ }^{26}$.

En los años más recientes comienzan a aparecer, además, valoraciones muy rigurosas del reto que suponen las $\mathrm{MAC}$ para la asistencia sanitaria del siglo $\mathrm{XXI}^{27}$ e iniciativas verdaderamente aperturistas como la serie de la revista $P e$ diatría Catalana, en la que, en los últimos años, se han incluido artículos para dar a conocer diferentes MAC y sus potenciales aplicaciones en el ámbito de la pediatría ${ }^{28}$.

En cuanto al conocimiento del uso de las MAC en el ámbito español hace unos años señalábamos que, excepto para el caso catalán, los datos eran prácticamente inexistentes ${ }^{29}$. Diversas encuestas de salud publicadas y/o llevadas a cabo en los últimos años han modificado en parte esta situación. Los datos

23. WINNINCK, T.A.: "Trends in attention to complementary and alternative medicine in the American medical literature», Health, 11 (2007), pp. 371-399.

24. Valtueña-Boraue, O.: «Sobre la homeopatía», Atención Primaria, 19 (1997), p. 384. FernándeZHerráez, E.: "Creer o no creer. Esa es la clave de la homeopatía», Atención Primaria, 21 (1998), pp. 593-596.

25. CALDERÓN, C.: "Médicos homeópatas y médicos de atención primaria: cómo se ven y cómo ven a sus pacientes. Resultados de una investigación cualitativa", Atención Primaria, 21 (1998), pp. 367-375. CALDERON, C.: «Medicina de familia y medicinas alternativas: propuesta de discusión», Atención Primaria, 24 (1999), pp. 176-177. Remon-MAsiP, J.; LiAnes-BARRAGAN, P.: «Medicinas complementarias en oncología», Atención Primaria, 39 (2007), pp. 5-6.

26. Borrell I CARrió, F.: «Homeopatía: creencia, praxis, pruebas», Medicina Clínica, 121 (2003), pp. 142-148. BORRELL I CARRIÓ, F.: «Medicinas complementarias y alternativas: algunas reflexiones desde la bioética", Atención Primaria, 35 (2005), pp. 311-313.

27. CAMinal-Homar, J.: «Medicinas complementarias o alternativas? Un dilema para el sistema público", Atención Primaria, 35 (2005), pp. 389-391. Caminal, J.; RodrígueZ, N.; Molina, J.L. y GRUPO DE INVESTIGACIÓN EN MCA: "Las medicinas complementarias y alternativas y su contribución al sistema sociosanitario: el qué, el porqué y el cómo», Atención Primaria, 38 (2006), pp. 409-412. Urios, C.; CAMinal, J.; RodríGUEZ, N.; PUigPelaT, F.: «Estrategias regulativas para las medicinas complementarias y alternativas: hacia un modelo de entendimiento en prácticas de salud", Atención Primaria, 38 (2006), pp. 574-576. ForCADEs, T.; CAMINAL, J.; RodríGUEZ, N.; GUTIÉRREZ, T. Y:. «Efecto placebo frente a efecto terapéutico en la práctica clínica y medicinas complementarias y alternativas", Atención Primaria, 39 (2007), pp. 99-102.

28. BARAIBAR, R.: «Medicines alternatives i complemetàries», Pediatria Catalana, 65 (2005), pp. 169 174. Albareda, C.; Molina, V.: "Coneguem les medicines complementàries», Pediatria Catalana, 65 (2005), p. 208. GIRAlT, I.; DALMAU, I.: "La medicina tradicional xinesa: un coneixement mil.lenari i cientific al servei de la salud del segle XXI», Pediatria Catalana, 65 (2005), pp. 264269. GoenagA, A.; Mínguez, D.: "Osteopatía. Qué és i quines aplicacions té en la pediatria», Pediatria Catalana, 66 (2006), pp. 77-80. SAZ, P.; ORTIZ, M.: "Medicina naturista en pediatia», Pediatria Catalana, 66 (2006), pp. 203-208.

29. Perdiguero, E.: "El fenómeno del pluralismo asistencial: una realidad por investigar», Gaceta Sanitaria, 18 (2004), pp. 140-145. 
conocidos hasta 2003 en el conjunto de Cataluña y en la ciudad de Barcelona apuntaban a una prevalencia de uso de las MAC que quedaba sensiblemente por debajo de lo que señalaban los datos de nuestro contexto europeo. Pero las características sociodemográficas de los usuarios coincidían con lo hallado en otros ámbitos: las mayores usuarias de MAC solían ser mujeres de edad media, con nivel de instrucción medio o alto y pertenecientes a los sectores sociales más acomodados. Este perfil de usuarios, no obstante, está comenzando a modificarse, al menos en los ámbitos en los que las MAC son una realidad más pujante, como ocurre en Estados Unidos ${ }^{30}$.

Los datos más recientes referentes a España siguen mostrando que las MAC son una realidad todavía incipiente, pero indican una importancia creciente, especialmente en el grupo de las mayores usuarias: mujeres de edad media con poder adquisitivo alto y/o medio y estudios universitarios, aunque las diferentes encuestas varían mucho en el modo en que han planteado el tema. Veámoslas con detalle.

Para el caso del conjunto del Estado español la Encuesta Nacional de Salud correspondiente a $2003^{31}$ halló que un 5,55 \% de los encuestados habían consumido productos de medicina alternativa en las dos semanas previas a la realización de la entrevista. Este porcentaje es, sin embargo, algo más relevante si nos referimos a las personas de edad media. Así los encuestados de entre 45 y 54 años habían recurrido a estos productos en un 8,14\% de los casos y los que tenían entre 55 y 64 años en un 7,92 \%. Si estas franjas etarias las restringimos a mujeres los porcentajes aun resultan más elevados. Un 11,66 \% de las mujeres de 45 a 64 años consumió productos alternativos en las dos semanas previas a la encuesta y en el caso de las de 55 a 64 el porcentaje se cifró en un 11, 24 \%. Los datos referidos al mayor o menor consumo de estos productos en función del tamaño del núcleo urbano no resultan demasiado esclarecedores, si bien, en el caso de las mujeres, este consumo fue más importante en municipios de más de 50.000 habitantes.

Sí resultan mucho más indicativos los datos que relacionan el consumo de productos alternativos y el nivel de estudios. Los poseedores de títulos universitarios hicieron uso de estos productos en un 7,60 \% de los casos mientras que los analfabetos o sin estudios sólo los consumieron en un 3,79 \%. Las mujeres con título universitario fueron las que alcanzaron un mayor porcentaje de consumo, un 9,61 \%. No hay grandes diferencias de uso de productos alternativos en función de la situación laboral, siendo muy similares los porcentajes entre los ocupados, los parados y los inactivos. Sí hay un claro gradiente, sin embargo, en el caso de la clase social del sustentador principal del núcleo familiar, siendo

30. En EEUU las MAC comienzan a ser utilizadas en igual medida por todos los segmentos sociodemográficos. COMPLEmentary and Alternative Medicine in the United STATeS: Op. Cit., p. 42 .

31. EnCUesta NACiOnal De SALud 2003, Madrid, Instituto Nacional de Estadística, (s.a.) Disponible en: http://www.msc.es/estadEstudios/estadisticas/encuestaNacional/encuestaNac2003/home. htm 
más frecuente el uso de productos alternativos en la clase social I (un 8,03 \%), descendiendo según lo va haciendo la clase social. Este gradiente resulta mucho más claro, como en los demás casos, en las mujeres, siendo las de clase social I las que presentaron un porcentaje más elevado de uso de productos alternativos, un $9,48 \%$. El uso de este tipo de preparados varió mucho por comunidades autónomas. A la cabeza se sitúa Cataluña (10, 60 \%), seguida del País Vasco $(8,47 \%)$ y la Comunidad Valenciana $(7,63 \%)$. En el extremo opuesto se sitúan Ceuta y Melilla (0,68 \%), La Rioja (1,39 \%) y Andalucía (1,98 \%). Nos encontramos por tanto con una situación que, si bien resulta modesta en la magnitud, se acomoda a las tendencias presentadas en otros ámbitos donde las MAC han sido mejor estudiadas y parecen tener una mayor entidad.

Además de estos datos nacionales contamos con otros referidos a diversas comunidades autónomas, que en sus encuestas de salud o en otros tipos de estudios poblacionales se han interesado por las MAC, aunque por la confección dispar de las preguntas que se emplean para interesarse por ellas impiden tener un panorama coherente de la prevalencia de uso.

La Encuesta de Salud de Asturias de $2002^{32}$, publicada a finales de 2003, nos proporciona datos sobre el uso de servicios sanitarios en las dos semanas previas a la encuesta. Un $0,3 \%(0,2 \%$ en el caso de los hombres y un $0,5 \%$ en el caso de de las mujeres) reconoció haber acudido al homeópata o acupuntor. La encuesta, además, nos informa que un $0,2 \%$-sin diferencias de género- acudió a un curandero/a o brujo/a. El apartado de "Otros profesionales», en el que podrían estar incluidos otros proveedores de MAC diferentes a homeópatas y acupuntores, arroja porcentajes del 1,9\% (1,2\% en los hombres y 1,9\% en las mujeres). Si nos atenemos a los datos que con seguridad se refieren a MAC se usaron más por parte de los que tenían entre 30 y 44 años (1 \%: 0,8\% en el caso de los hombres, $1,1 \%$ en el caso de las mujeres). La encuesta, además, nos informa de que el uso de homeopatía/acupuntura sólo se produjo en el caso de los hombres en la zona central del Principado de Asturias, mientras que estas opciones fueron más utilizadas en la zona rural en el caso de las mujeres. Al contrario de lo que suele ser la norma, los usuarios de homeopatía y acupuntura pertenecían sobre todo a las clases sociales IV y V.

La I Encuesta de Salud de Castilla-La Mancha, realizada en $2002^{33}$ también se interesó por el uso de las MAC. Los datos que arrojó este estudio nos indican la poca relevancia de las mismas en esta región hace unos años. En el año previo a la entrevista sólo un 0,3 \% de los entrevistados acudió al homeópata, un 0,2 $\%$ al osteópata, un $0,2 \%$ al naturista, y un $0,3 \%$ al acupuntor. Sin embargo, los autores del estudio señalan que quienes utilizaron están opciones lo hicieron en torno a tres veces por año. También suponen porcentajes ínfimos el recurso

32. Encuesta de Salud para Asturias 2002, Oviedo, Consejería de Salud y Servicios Sanitarios. Gobierno del Principado de Asturias, 2003. Disponible en: http://www.princast.es/servlet/page?_pageid=2727\&_dad=portal301\&_schema=PORTAL30

33. Encuesta de Salud de Castilla la Mancha 2002, Toledo, FISCAM, (s.a.). Disponible en: http://www.jccm.es/sanidad/fiscam/cursos.php?idTituloDef=38 
al curandero $(0,3 \%)$ o a los gabinetes de estética como recurso sanitario $(0,6$ $\%)$. Para este último caso, no obstante, se señala que las mujeres que lo usaron acudieron más de cinco veces durante el año ${ }^{34}$. Más visitados fueron los herbolarios, pues un $1,3 \%$ de los entrevistados afirmaron haberlos utilizado durante los 12 meses previos.

Cuatro años después ${ }^{35}$ la situación en Castilla-La Mancha quedaba configurada de la siguiente manera: los porcentajes de personas que acudían al homeópata y al osteópata eran similares a los de el año 2002 y el recurso al curandero había descendido $(0,1 \%)$. Sin embargo se había producido un ascenso considerable en la utilización de la acupuntura y de la naturopatía, que presentaban porcentajes de un $0,7 \%$ y $0,8 \%$ respectivamente. Aunque el aumento más acusado fue el de los gabinetes de estética que fueron utilizados con fines sanitarios por un 4,6\% de la población, alcanzando el 10,6\% en el grupo de edad comprendido entre los 16 y 24 años. En todos los casos salvo en el de la consulta a homeópatas, en la que el porcentaje fue igual para ambos sexos, fueron las mujeres las que más utilizaron todas las MAC.

Para Castilla y León los últimos datos autonómicos provienen de la Encuesta Regional de Salud $2003^{36}$, que en realidad fue una ampliación de la Encuesta Nacional de Salud 2003, por lo que las preguntas son las mismas que las realizadas en el ámbito nacional. En este caso, un 4,16 \% de los encuestados afirmó haber consumido productos de medicina alternativa en las dos semanas previas. Las mujeres, como en el conjunto del país, presentaron un porcentaje de uso más alto $(6,1 \%)$ que los hombres $(2,17 \%)$. Y dentro de ellas fueron las pertenecientes al grupo de edad media (grupo de 45 a 54 años) las que presentaron un mayor porcentaje de uso $(9,57 \%)$.

$\mathrm{Al}$ igual que ocurría en todo el territorio nacional son los universitarios los que más consumen productos alternativos $(5,62 \%)$ frente al grupo de analfabetos o sin estudios que son los que menos los consumen (3,04\%). También se cumple en el caso de Castilla y León el gradiente de clase social, siendo más frecuente el consumo de productos alternativos según se asciende en la escala social. Si atendemos a la situación laboral los datos arrojan un mayor porcentaje de consumo de productos alternativos en el caso de los parados $(7,42 \%)$, bastante por delante de los ocupados $(4,02 \%)$ o los inactivos $(4,16$ $\%$ ). El porcentaje es más rotundo en el caso de las mujeres paradas, con un 13,7 $\%$ de consumidoras de productos alternativos. En el caso de Castilla y León el

34. La encuesta no explica, lamentablemente, con mayor detalle cuáles fueron los «motivos de salud» que llevaron a los gabinetes de estética, circunstancia que hubiera sido muy interesante conocer ya que esta instancia no suele ser considerada por las encuestas de salud y otros estudios poblacionales.

35. Encuesta de Salud de Castilla La Mancha 2006, Toledo, FISCAM, (s.a.).Disponible en: http://www.jccm.es/sanidad/FISCAM/ensa06/ensa06.html

36. EnCUeSTA Regional De SALud 2003, Valladolid, Consejería de Sanidad. Junta de Castilla y León, 2005. Disponible en:

http://www.sanidad.jcyl.es/sanidad/cm/institucion/tkContent?pgseed=1177272334986\&idCo ntent $=181828 \&$ locale $=$ es_ES\&textOnly $=$ false 
recurso a productos alternativos ocurre, sobre todo, en municipios de más de 50.000 habitantes.

La Encuesta de Salud de Canarias de $2004^{37}$ también nos aporta información relativa al uso de MAC y curanderos. Un 7,61 \% de los encuestados mayores de 16 años en Canarias acudieron en alguna ocasión en su vida al homeópata, osteópata o naturista. El acupuntor fue utilizado por un 2,98 \% de los preguntados, y el curandero fue consultado en un $3,72 \%$ de los casos. Las mujeres de 30 a 44 años (16,93\%), las de 16 a 29 años (16,38 \%) y los hombres de 30 a 44 años $(16,67 \%)$ fueron los grupos que más usaron, alguna vez en su vida, alguna de las opciones que la Encuesta de Salud de Canarias consideró «medicina alternativa».

También se preguntó por el uso de "productos de medicina alternativa (homeopática, naturista, etc.)» durante las dos semanas previas a la realización de la entrevista, en el marco de una pregunta dirigida a conocer el consumo de medicamentos por parte de la población. Pero a la hora de presentar los resultados esta categoría se colapsó con otras referidas al consumo de laxantes, medicamentos para la alergia, medicamentos para procesos reumáticos, medicamentos hormonales sustitutivos y medicamentos para adelgazar; por lo que no podemos hacernos una idea de a qué corresponde el 11,65\% de encuestados que afirmó haber consumido alguno de estos productos.

En el caso de la Comunidad Valenciana, la Encuesta de Salud de 2000-200138 se había interesado por la posible preferencia de los valencianos por las MAC, pero sin valorar su uso real. La Encuesta de Salud de $2005^{39}$ realizada en el territorio valenciano, y publicada en 2007, incluyó una cuestión sobre la mayor frecuencia de utilización de servicios sanitarios en los últimos 12 meses, en la que se daba la oportunidad de escoger la opción "Otros métodos (naturistas...)", diferenciando entre servicios públicos o privados, lo cual para este tipo de proveedores resulta especialmente sorprendente. Con este tipo de cuestión resulta complicado hacerse una idea de la magnitud de uso de las MAC en tierras valencianas y no resulta extraño que sólo un 2,9\% de los encuestados reconocieran que usaban con mayor frecuencia esos "otros métodos» en el año previo. Curiosamente un 1,2 \% señaló que lo usaba en el ámbito público, por lo que suponemos que bajo esa rúbrica se consideraron otras opciones terapéuticas que la encuesta no nos permite entrever. En todo caso fueron los usuarios de edad media (3\%), con estudios secundarios $(3,5 \%)$ o universitarios $(3,5 \%)$, con ingresos superiores a 6.000 euros al mes $(17,2 \%)$, mujeres $(3,9 \%)$ y los

37. EnCUeSTA DE SALUd de CANARIAS 2004, Instituto Canario de Estadística. Servicio Canario de Salud, (s.a.). Disponible en: http://www.gobcan.es/istac/estadisticas/salud_2004/salud2004.html

38. EnCUesta de SALud de la COMUnidad VAlenciana 2000-2001, Valencia, Conselleria de Sanitat. Generalitat Valenciana, 2002. Disponible en:

http://www.san.gva.es/cas/comun/encuesta/documentoscompletos.htm

39. EnCuesta De SAlud De la Comunidad Valenciana 2005, Valencia, Consellería de Sanitat. Generalitat Valenciana, 2007. Disponible en:

http://www.san.gva.es/cas/comun/encuesta/documentoscompletos.htm 
pertenecientes a la clase social I (4,8\%) los que con mayor frecuencia recurrieron a estas opciones.

Los datos más recientes sobre el uso de las MAC a los que hemos tenido acceso han sido publicados a lo largo de 2007. Así, en el estudio de modos de vida en Vitoria-Gasteizt $2006^{40}$, también se aportan datos sobre las MAC. Un 6 $\%$ de los encuestados en la capital alavesa acudieron a medicinas alternativas en el año previo siendo, como es habitual, mayor el porcentaje de mujeres $(7 \%)$ que el de hombres (5\%). Los grupos de edad que aporta el estudio son muy grandes y no hay diferencias entre ellos: un $7 \%$ de las mujeres entre 25 y 64 años utilizó las medicinas alternativas en los últimos doce meses, e igual porcentaje presentaron los hombres y mujeres de 65 y más años.

La Enquesta de Salut de Catalunya $2006^{41}$, cuyos datos todavía sin elaborar ya están disponibles, arroja para el caso de las MAC unos resultados un tanto sorprendentes, pues aunque suponen un aumento de la relevancia de las MAC con respecto a los datos previos de 1994 y $2002^{42}$, este aumento es muy poco importante y parece no ser coherente con el esfuerzo regulador de las MAC acometido por la Generalitat de Catalunya ${ }^{43}$.

La utilización de medicamentos que pueden ser considerados dentro de las MAC es muy poco importante entre los catalanes de 15 años o más. Sólo un $1,1 \%$ de los encuestados tomó medicamentos homeopáticos recetados por un médico, un $0,1 \%$ lo hizo aconsejado en la farmacia ${ }^{44}$, y un 0,3 \% los tomó por iniciativa propia. En el caso de las plantas medicinales adquiridas en una farmacia un $0,6 \%$ las tomó con receta médica, un 0,4\% las tomó por consejo obtenido en la propia farmacia, y un $1 \%$ por iniciativa propia. En el caso de plantas medicinales adquiridas en herboristerías un $1 \%$ de los encuestados reconoce haberlas tomado siguiendo prescripción médica, un 0,3 \% por consejo de la farmacia, y un 3,2 \% por propia iniciativa. En todos lo casos, comparativamente, las mujeres tomaron más estos medicamentos que los hombres.

En lo que se refiere a la utilización de proveedores de MAC por parte de los catalanes de 15 años o más los datos señalan que en los 12 meses previos a la entrevista acudió al osteópata un 2,8 \% de los entrevistados, al homeópata un 2 $\%$, al naturista un 2,2 \% y al acupuntor un 1,8 \%. En todos los casos las mujeres usaron más estas alternativas que los hombres, y fueron las mujeres de edad media (de 45 a 64 años) las mayores usuarias, excepto en el caso del recurso a

40. Estudio De Modos DE VidA en Vitoria-Gasteiz 2006. «Aspectos relacionados con la salud, según el género", Vitoria, Ayuntamiento de Vitoria-Gasteiz, 2007.

41. Enquesta de Salut de Catalunya 2006. Barcelona, Departament de Salut, Generalitat de Catalunya, 2007. Disponible en:

http://www.gencat.net/salut/depsan/units/sanitat/html/ca/plasalut/doc11898.html

42. Perdiguero, E.: «El fenómeno del pluralismo asistencial: una realidad por investigar», Op. cit., pp. $140-145$.

43. Decret $31 / 2007$ de 30 gener, pel qual es regulen les condicions per a l'exercici de determinades teràpies naturals. Diari Oficial de la Generalitat de Catalunya, 1-12-2007, pp. 3758-3765.

44. Este es el item que aparece en la tabla publicada, aunque es de suponer que se referirá a consejo obtenido en la propia herboristería. 
la osteopatía. Esta terapia manipulativa fue usada en un mayor porcentaje $(6,9$ $\%)$ por las mujeres de 75 años y más. Un porcentaje todavía más pequeño (0,3 \%) supone el uso de otras terapias manipulativas como el quiromasaje o la quiropráctica.

El estudio catalán también se ha ocupado de inquirir por las consultas realizadas en los 15 días previos a la realización de las entrevistas. Todas las MAC consideradas -osteópata, homeópata, naturista, acupuntor- fueron utilizadas en similar porcentaje, un ínfimo $0,2 \%$, siendo levemente más utilizadas por las mujeres en casi todos los casos.

Los datos correspondientes a los menores (de 0 a 14 años) muestran también porcentajes de usos de las MAC en los 12 meses previos muy poco relevantes. El osteópata fue utilizado en un 0,7 \% de los casos, el homeópata en un 3,1 \%, el naturista en un 0,8 \%, y el acupuntor en un 0,3\%. En este caso no se da la preponderancia femenina. Los niños fueron los que usaron más todas las opciones, excepto en el caso de la homeopatía a la que recurrieron por igual niños y niñas $(3,1 \%)$. El quirosamaje y la quiropráctica fue una alternativa usada sólo por el $0,4 \%$ de los niños y niñas encuestados.

Además de las encuestas de salud podemos citar algunos otros ejemplos de pequeños estudios que nos dan alguna idea de la prevalencia de uso de las MAC en España y que nos aportan algunas claves sobre los motivos de uso de las mismas.

En primer lugar, el avance de resultados de la $2^{\text {a }}$ edición de la Encuesta Social Europea ${ }^{45}$ resalta la buena valoración que los españoles tienen del sistema sanitario, cercana al grupo de países que mejor valoran su sanidad. Por el contrario España se encuentra lejos de los países europeos que más recurren a las MAC: Austria, Noruega, Suiza y Dinamarca.

Las MAC son, como ocurre en otros muchos países, un recurso ampliamente utilizado para diversas enfermedades crónicas o de difícil curación como el dolor de espalda ${ }^{46}$, el cáncer ${ }^{47}$, o las enfermedades reumáticas ${ }^{48}$. Así, en nuestro

45. Los Españoles en Europa. Avance de los Resultados de la $2^{a}$ Edicion de la EnCuesta SoCIAL EUROPE, Madrid-Barcelona, Ministerio de Educación y Ciencia. Universitat Pompeu Fabra. Instituto Demoscopia, 2006.

46. Sherman, K.J.; Cherkin, D.C.; CONNelly, M.T.; ERro, J.; SAVETSKY, J.B., DAVIS, R.B.; EISENBERG, D.M.: "Complementary and alternative medical therapies for chronic low back pain: What treatments are patients willing to try?», BMC Complementary and Alternative Medicine, 4 (2004), p. 9 (http://www.biomedcentral.com/1472-6882/4/9).

47. García-Nieto, A.; Capote-Huelva, F.j.; Fernández-Valle, M.C.; Gil-García, J.L.: "CAM en pacientes con neoplasias linfoides», Medicina Clínica, 123 (2004), p. 676. IDOYAGA MOLINA, A.; LUXARDO, N.: «Medicinas no convencionales en cáncer», Medicina (Buenos Aires), 65 (2005), pp. 390-394.

48. Hernández-Ríos, G.; DurÁn-BARRAGÁn, S.; RAmos-Remus, C.: "Los tratamientos no convencionales y las terapias alternativas en la era de grandes avances terapéuticos en reumatología", Reumatología Clínica, 2 (2006), pp. 161-163. ÁlvareZ-HernándEZ, E.; CASASOlA-VARGAS, J.C.; Lino-PÉREZ, L.; BuRgos-VARGAS, R.; VÁzQuez-MELlado, J.: «Frecuencia de uso de medicinas complementarias y alternativas en sujetos que acuden por primera vez al servicio de reumatología. Análisis de 800 casos», Reumatología Clínica, 2 (2006), pp. 183-189. 
ámbito, según datos recientes, se reconoció el uso de $\mathrm{MAC}$ en algún momento de la evolución de la enfermedad en un $77 \%$ de un grupo de enfermos con neoplasias linfoides ${ }^{49}$, en un $37 \%$ de un grupo de aquejados de hepatitis C crónica $^{50}$, en un $26 \%$ de un grupo de pacientes con enfermedad inflamatoria intestinal ${ }^{51}$, en un $48 \%$ de pacientes con fibromialgia ${ }^{52}$, y en un $30 \%$ de un grupo de enfermos aquejados de padecimientos somatoformes ${ }^{53}$.

\section{ALGUNAS REFLEXIONES SOBRE EL USO DE LAS MAC POR PARTE DE LAS MUJERES}

Como hemos señalado durante de todo el artículo, las mujeres son las principales usuarias de las MAC, sin embargo, se conoce poco sobre los motivos por los cuales esto sucede ${ }^{54}$. En la mayoría de los estudios el sexo se considera sólo un rasgo sociodemográfico que contribuye a caracterizar el perfil de los usuarios de estas medicinas, sin aportar ninguna explicación sobre las causas de esta distribución, o se interpreta como parte de la alta frecuentación de las mujeres de todos los servicios sanitarios. Esta situación se ha intentado paliar, en los últimos años, mediante trabajos realizados exclusivamente con mujeres $^{55}$, sin embargo, en estos casos, la relación femenina con las MAC se presenta a través de datos cuantitativos -descriptivos- sin ahondar en los motivos que producen estas diferencias de género en la utilización.

Por ello, aunque parece paradójico, a pesar de que las MAC se están convirtiendo en un recurso importante en la gestión de la salud para las mujeres, son una realidad muy poco explorada ${ }^{56}$. Existen múltiples lagunas en el cono-

49. García-Nieto, A.; Capote-Huelva, F.j.; Fernández-Valle, M.C.; Gil-García, J.L: Op. cit., p. 676.

50. Bruguera, M.; Barrera, J.M.; AmpurdanéS, S.; Forns, X.; SÁnCheZ-TAPIAS, J.M.: «Utilización de las medicinas alternativas y complementarias por los pacientes con hepatitis C crónica", Medicina Clínica, 122 (2004), pp. 334-335.

51. García-Planella, E.; Marín, L.; Domènech, E.; Bernal, I.; Mañosa, M.; ZabanA, Y.; Gassull, M.A.: "Utilización de medicinas alternativas y consumo de drogas por pacientes con enfermedad inflamatoria intestinal», Medicina Clinica, 128 (2007), pp. 45-48.

52. JunYent-PriU, M.; CAMP-HERRERO, J.; FernÁNDEZ-SOlÁ, J.: «Utilización de la medicina alternativa y complementaria en la fibromialgia», Medicina Clínica, 124 (2005), p. 397.

53. GaRCíA-CAMPAYO, J.; SÁNZ-CARRILlO, C.: "The use of alternative medicines by somatoform disorder patients in Spain", British Journal of General Practice, 50 (2000), pp. 487-488.

54. ADAMS, J.; EASTHOPE, G.; SIBBRITT, D.: «Exploring the relationship between women's health and the use of complementary and alternative medicine", Complementary Therapies in Medicine, 11 (2003), pp. 156-158.

55. Cushman, L.F.; Wade, C.; Factor-Litvak, P.; KronenberG, F.; Firester, L.: «Use of complementary and alternative medicine among African-American and Hispanic women in New York city», Journal of American Medical Women's Association, 54 (1999), pp. 193-195. ADAMS, J.; SIBBRITT, D.; EASTHOPE, G.; YOUNG, A.: «The profile of women who consult alternative health practicioners in Autralia", Medical Journal of Australia, 179 (2003), pp. 297-300. DAWN, M.U.; CHYU, L.: "Use of complementary and alternative medicine among American women", Women's Health Issues, 15 (2005), pp. 5-13.

56. Aikins, P.; KRONENBERG, F.; WADE, C.: "Complementary and alternative medicine in women's health. Developing a research agenda", Journal of Nurse-Midwifery, 44 (1999), pp. 192-204. ADAMS, J.; EASTHOPE, G.; SibBritT, D.: Op. Cit., pp. 156-158. FlesCH H.: «Silent voices: women, 
cimiento de esta relación como, por ejemplo, el tipo de consumo que de las MAC hacen las mujeres, la variación en las tendencias de consumo a lo largo del tiempo y las terapias concretas utilizadas, el grado de automedicación con productos naturales o hasta qué punto las mujeres, como cuidadoras, tratan con este tipo de productos/terapias al resto de miembros de la familia.

Esta ausencia de trabajos puede ser interpretada como un signo de la invisibilidad ${ }^{57}$ que padecen las mujeres en medicina y que se ha mostrado ya en otros ámbitos relacionados con el estudio de sus padecimientos y su comportamiento frente a la enfermedad ${ }^{58}$. A pesar de ello pueden plantearse algunas hipótesis, unas relacionadas con rasgos de los usuarios de las MAC y otras con las propias características de estas terapias.

El perfil de los usuarios, además de su caracterización demográfica, se dibuja en función de otras características relacionadas con sus creencias sobre la salud y la enfermedad, el tipo de padecimientos para el que utilizan las MAC y su relación con la medicina científico-occidental.

Los usuarios de MAC han sido definidos como personas que se consideran a sí mismas como creativas e innovadoras y que presentan un alto grado de autonomía y un rol activo en el cuidado y mantenimiento de su salud. Estas características junto la importancia que otorgan al papel de la mente y lo espiritual en el mantenimiento y restablecimiento de la salud hacen que consideren a las MAC más congruentes con su filosofía holista de la salud ${ }^{59}$.

En cuanto al estado de salud, en general, las MAC son utilizadas por personas que presentan multitud de síntomas físicos y tienen mala salud -autopercibida- ${ }^{60}$. En cuanto al tipo de padecimiento por el que las utilizan, son frecuentes las enfermedades crónico-degenerativas y de difícil curación, o controvertidas dentro de la propia medicina ${ }^{61}$. Tanto unas como otras son más prevalentes en

complementary medicine and co-optation change», Complementary Therapies in Clinical Practice, 13 (2007), pp. 166-173.

57. También existe un sesgo en el estudio de las MAC por el que se construye la imagen de la mujer como consumidora y no como proveedora, invisibilizando el hecho de que la mayoría de proveedores de estas terapias son mujeres. Otro sesgo se produce por la consideración que se ha hecho de las diferentes modalidades de práctica de las MAC y su división por sexos, ya que se ha presentado al hombre como terapeuta de modalidades consideradas científicas (homeopatía o quiropráctica), lucrativas y que se realizan a tiempo completo mientras que las mujeres han sido relacionadas con otras como la aromaterapia, los masajes o la reflexología, que se consideran más relacionadas con la belleza, la armonía y lo místico, y como extensión de su responsabilidad de cuidado en la esfera doméstica y no como actividades profesionales. FLESCH H.: Op. cit., pp. 167.

58. VAlLs-LlobeT, C.: Mujeres invisibles para la medicina, Barcelona, Mondadori, 2006.

59. AsTIN, J.A.: "Why patients use alternative medicine? Results of a national study», JAMA, 279 (1998), pp. 1548-1553.

60. SIROIS, F.M.; GICK, M.L.: "An investigation of health beliefs and motivations of complementary medicine clients», Social Science and Medicine, 55 (2002), pp. 1025-1037.

61. Dentro de ellas se encuentran múltiples síndromes considerados funcionales como el síndrome de fatiga crónica, la cistitis intersticial, la fibromialgia, el síndrome de sensibilidad química múltiple, el síndrome de intestino irritable y otros padecimientos como los trastornos somatoformes. En todos estos casos existe discrepancia, en cuanto al origen y posible tratamiento, 
las mujeres. Por otro lado, hay que tener en cuenta que muchas de estas enfermedades y síntomas padecidos por las mujeres, como por ejemplo el dolor crónico, han recibido poca atención por parte de la medicina científico-occidental, lo que hace necesaria la búsqueda de otras alternativas para su tratamiento.

La relación de los usuarios de las MAC con la medicina científico-occidental es ambigua puesto que, aunque en muchos casos presentan desconfianza y consideran que es un recurso ineficaz para resolver un problema de salud concreto, por otro lado, son hiperfrecuentadores de todos los servicios sanitarios y utilizan las MAC simultáneamente con la medicina científico-occidental y no sólo cuando ésta falla o tiene efectos adversos ${ }^{62}$.

En muchos casos refieren insatisfacción con la interacción con los sanitarios, en especial con los médicos, por diversas razones entre las que se encuentran, fundamentalmente, los problemas de comunicación y la limitada capacidad de negociación y decisión sobre las actuaciones médicas que afectan a su salud y su bienestar ${ }^{63}$. Esta situación ha sido ampliamente estudiada en el contexto de la salud de las mujeres, sobretodo en referencia a la medicalización de los procesos propios del ciclo vital femenino ${ }^{64}$.

Además, en la negociación de algunos padecimientos como por ejemplo la fibromialgia o los trastornos somatoformes, que también son mucho más frecuentes en mujeres, se produce una deslegitimación/descrédito de los síntomas $y$, a la vez, de la persona que los padece. En ese sentido, las MAC ofrecen, además de alternativas pragmáticas, la posibilidad de restaurar la legitimidad de la mujer y aumentar su capacidad de negociación/decisión sobre su padecimiento. Las MAC en este sentido pueden ser entendidas como una forma de resistencia frente a hegemonía médica y las desigualdades en salud a las que se enfrentan las mujeres ${ }^{65}$.

Por último, las MAC presentan características que podrían relacionarse con la frecuencia de uso por parte de las mujeres. Además de que se consideren como naturales, inocuas y sin efectos adversos ${ }^{66}$, algunos trabajos plantean que las MAC proporcionan un estilo más femenino y feminista de cuidado que la medicina convencional ${ }^{67}$ ya que devuelven el control del cuerpo a la persona

entre médicos y pacientes e incluso entre profesionales de diferentes especialidades médicas. TOSAL, B.: "Síndromes en femenino. El discurso médico sobre la fibromialgia», Feminismo/s (en este número).

62. Adams, J.; SibBritT, D.; EASthope, G.; YounG, A.: Op. cit., p. 299.

63. Brown, J.B.; CARroll, J.; BoON, H.; MarmoreO, J.: «Women's decision-making about their health care: views over the life cycle», Patient Education and Counselling, 48 (2002), pp. 225-231. SIROIS, F.M.; GICK, M.L.: Op. cit., p. 1027.

64. RIESSMAN, C.K.: «Women and medicalization. A new perspective», en R. Weitz (Ed.): The politics of women's bodies: Sexuality, appearance, and behavior, New York, Oxford University Press, 1998, pp. 46-63. ESTEBAN, M.L.: Re-producción del cuerpo femenino. Discursos y prácticas acerca de la salud, Donostia, Tercera Prensa, 2001.

65. Flesh, H.: Op. Cit., p. 170.

66. Brown, J.B.; CARroll, J.; BoOn, H.; Marmoreo, J.: Op. cit., p. 228.

67. SCOTT, A.: "Homeopathy as a feminist form of medicine», Sociology of Health and Illness, 20 (1998), pp. 191-214. 
-una de las reivindicaciones típicamente feministas en relación a la medicina científico-occidental- y permiten una relación más igualitaria entre el terapeuta y el enfermo.

\section{CONCLUSIONES}

Como hemos podido comprobar, las MAC tienen, aún, una importancia menor como recurso para enfrentar los problemas de salud en nuestro contexto, aunque con el paso del tiempo su presencia ha ido aumentando. Los datos, aunque parciales ${ }^{68}$, nos muestran la misma tendencia que en el resto del mundo en cuanto a los motivos de uso -enfermedades crónicas y de difícil curación pero, también, aquellas más controvertidas dentro del ámbito de actuación médica- y los perfiles sociodemográficos de los usuarios -mujeres, de clase alta y con estudios-.

En el caso de las mujeres, a pesar de ser reconocida su alta prevalencia de uso, no se ha indagado suficientemente en los motivos por los cuales utilizan las MAC. Este hecho tiene que ver con la invisibilización a la que, como en otros muchos ámbitos de la medicina, están sometidas las mujeres. Así, las hipótesis sobre su mayor recurrencia a las MAC están basadas, en muchas ocasiones, más en proyecciones teóricas que en datos empíricos. No obstante, la hipótesis de la resistencia a la medicalización de muchos procesos femeninos y la mayor capacidad de negociación con este tipo de terapeutas, junto con la alta prevalencia de padecimientos para los que no existen soluciones en el ámbito de la medicina científico-occidental, pueden explicar, al menos en parte, los motivos por los que las mujeres recurren más a ese tipo de instancias terapéuticas.

68. Los porcentajes de uso en función de la enfermedad no se han obtenido de muestras de población general sino de colectivos de enfermos. 